\title{
The ColRS system is essential for the hunger response of glucose-growing Pseudomonas putida
}

\author{
Marta Putrinš, Andres Ainelo, Heili Ilves and Rita Hõrak*
}

\begin{abstract}
Background: The survival of bacteria largely depends on signaling systems that coordinate cell responses to environmental cues. Previous studies on the two-component ColRS signal system in Pseudomonas putida revealed a peculiar subpopulation lysis phenotype of colR mutant that grows on solid glucose medium. Here, we aimed to clarify the reasons for the lysis of bacteria.

Results: We present evidence that the lysis defect of $P$. putida colR mutant is linked to hunger response. A subpopulation prone to lysis was located in the periphery of bacterial cultures growing on solid medium. Cell lysis was observed in glucose-limiting, but not in glucose-rich conditions. Furthermore, lysis was also alleviated by exhaustion of glucose from the medium which was evidenced by a lower lysis of central cells compared to peripheral ones. Thus, lysis takes place at a certain glucose concentration range that most probably provides bacteria a hunger signal. An analysis of membrane protein pattern revealed several hunger-induced changes in the bacterial outer membrane: at glucose limitation the amount of OprB1 channel protein was significantly increased whereas that of OprE was decreased. Hunger-induced up-regulation of OprB1 correlated in space and time with the lysis of the colR mutant, indicating that hunger response is detrimental to the colR-deficient bacteria. The amount of OprB1 is controlled post-transcriptionally and derepression of OprB1 in glucose-limiting medium depends at least partly on the carbon catabolite regulator protein Crc. The essentiality of ColR in hunger response can be bypassed by reducing the amount of certain outer membrane proteins. In addition to depletion of OprB1, the lysis defect of colR mutant can be suppressed by the down-regulation of OprF levels and the hindering of SecB-dependent protein secretion.

Conclusions: We show that Pseudomonas putida growing on solid glucose medium adapts to glucose limitation through up-regulation of the sugar channel protein OprB1 that probably allows enhanced acquisition of a limiting nutrient. However, to survive such hunger response bacteria need signalling by the ColRS system. Hence, the ColRS system should be considered a safety factor in hunger response that ensures the welfare of the cell membrane during the increased expression of certain membrane proteins.
\end{abstract}

\section{Background}

Most bacteria live in constantly changing and often nutritionally limiting environments. The success of bacteria in such conditions depends on their ability to sense the nutritional status of the environment and respond appropriately by reprogramming their gene expression and cell metabolism. For instance, nutrient depletion triggers starvation response that involves the stress-specific sigma factor RpoS and results in drastic changes in gene expression and finally arrests cell

\footnotetext{
* Correspondence: rhorak@ebc.ee

Institute of Molecular and Cell Biology, University of Tartu, Riia 23, Tartu, Estonia
}

() 2011 Putrinš et al; licensee BioMed Central Ltd. This is an Open Access article distributed under the terms of the Creative Commons Attribution License (http://creativecommons.org/licenses/by/2.0), which permits unrestricted use, distribution, and reproduction in any medium, provided the original work is properly cited. growth and division [1]. Bacteria can also discriminate between nutrient-rich and nutrient-poor conditions and respond to nutrient limitation through a regulated nutrient-specific hunger response [2]. Hunger response, activated when the growth rate of a bacterial population decreases due to limited acquisition of nutrients, essentially differs from the starvation response. While the starvation response prepares a cell population for survival in a nutrient-depleted environment, the hunger response improves the ability of bacteria to grow under nutrient-poor conditions [3]. The most obvious bacterial physiological response to low nutrient levels is the enhancement of scavenging ability for the limiting 
nutrient $[2,4]$. For instance, if $E$. coli is cultivated in glucose-limited chemostat, its permeability to glucose is increased through up-regulation of several outer membrane porins and high-affinity cytoplasmic membrane transporters [5-8]. However, as the rpoS gene was not induced in these conditions, hunger-induced changes should be considered distinct from stationary phase response [8]. Importantly, the mutants that are defective in some hunger-induced transporter have reduced fitness in nutrient-poor conditions $[5,9]$.

Hunger response has been studied by cultivation of bacteria in chemostat which allows a long-term and almost steady-state growth in nutrient-limiting conditions [2]. However, liquid batch cultures of bacteria also transiently experience a nutrient-limited period just before the exhaustion of the carbon source from the medium. Bacteria that grow on solid surfaces, e.g. on agar plates, encounter specific complications of nutrient acquisition, as during consumption of growth substrates niches with different nutrient level develop, which in turn results in a cellular differentiation and an increase in population heterogeneity [10]. The main difference between growth conditions of bacteria in liquid and on solid media is the development of nutrient concentration gradients during the growth on solid medium. This may significantly influence bacterial responses, as has been illustrated by the spatially and temporally different expression of a reporter gene in Bacillus subtilis $[11,12]$. Similarly, nutrient gradients that develop in other types of structured multicellular bacterial consortia, e.g. in biofilms, cause considerable physiological heterogeneity [13]. For example, the $P$. aeruginosa biofilm was reported to contain subpopulations with different metabolic activity: metabolically active bacteria were found in the upper layer and cells with low metabolic activity in the interior of the biofilm. Interestingly, such metabolic heterogeneity resulted in different adaptation responses as well as varied tolerance to antibiotics among subpopulations [14]. Thus, nutrient gradients strongly affect the behaviour of bacterial population on solid media.

Pseudomonas putida is a metabolically versatile bacterium widely distributed in the nature $[15,16]$. The comparison of genomes of P. putida and other Pseudomonas bacteria revealed 3,708 shared coding sequences [17]. The genes of the ColRS two-component signal transduction pathway are highly conserved in all Pseudomonas species [18] and growing evidence shows that the absence of the ColRS two-component system leads to several defects in different pseudomonads. Deficiency in the ColRS system results in the lowered root colonization ability of P. fluorescens $[19,20]$ and the attenuated virulence of $P$. aeruginosa [21]. Several ColRS-deficiency related phenotypes are also reported for $P$. putida, including down-regulation of stationary phase mutational processes [22], lowered phenol tolerance [23] and an increased susceptibility of cells to divalent metal ions [24]. We observed recently that under certain circumstances, the ColRS system is essential for the viability of $P$. putida. The colR-deficient $P$. putida displays a serious defect on the solid glucose medium where a subpopulation of bacteria lyses as evidenced by the release of cytoplasmic proteins and chromosomal DNA [25]. Intriguingly, the lysis of colR mutant occurs only on glucose and not on any other carbon source. Flow cytometry of propidium iodide-stained cells showed that even though most of the glucose-grown colR-deficient cells were indistinguishable from the wild-type, a minor subpopulation of cells had a seriously damaged membrane permeable to propidium iodide [25].

In the current study we took different approaches to understand i) why only a subpopulation of colR mutant lyses and ii) why the cell lysis occurs only on glucose medium. We identified several mutations that suppressed the lysis phenotype of colR-deficient bacteria and indicated that lysis is caused by hunger-induced changes in the outer membrane composition, including the accumulation of sugar channel protein OprB1. We showed that the degree of hunger response and the lysis of bacteria depend on glucose gradient building up in solid medium during the growth of bacteria - both traits were significantly elevated within the peripheral subpopulation of the colR-deficient strain. We conclude that ColRS system is needed for the proper response of bacteria to glucose limitation and contributes to the maintenance of membrane homeostasis under the increased expression of nutrient scavenging systems.

\section{Methods}

\section{Bacterial strains, plasmids, and media}

The bacterial strains and plasmids we used are described in Table 1. All experiments were conducted with derivatives of $P$. putida strain PaW85 [26] which is isogenic to fully sequenced KT2440 [27]. Bacteria were grown on Luria-Bertani (LB) medium [28] or on minimal medium [29] containing either $0.2 \%$ glucose, $0.2 \%$ Na-benzoate or $0.2 \%$ gluconate. Some experiments were performed with bacteria grown on media with glucose concentrations of 0.4 and $0.8 \%$. To enhance the lysis of the colR mutant, in some experiments $1 \mathrm{mM}$ phenol was added into the solid minimal medium. Congo Red at $0.0005 \%$ was added to the medium for visual evaluation of cell lysis. When selection was necessary, the growth medium was supplemented with ampicillin $(100 \mu \mathrm{g} / \mathrm{ml})$, streptomycin $(20$ $\mu \mathrm{g} / \mathrm{ml})$ or gentamicin $(10 \mu \mathrm{g} / \mathrm{ml})$ for $E$. coli and with carbenicillin $(1500 \mu \mathrm{g} / \mathrm{ml})$, kanamycin $(50 \mu \mathrm{g} / \mathrm{ml})$, streptomycin $(300 \mu \mathrm{g} / \mathrm{ml})$, tetracycline $(20 \mu \mathrm{g} / \mathrm{ml})$ or gentamicin $(10 \mu \mathrm{g} / \mathrm{ml})$ for $P$. putida. P. putida was 
Table 1 Bacterial strains and plasmids

\begin{tabular}{|c|c|c|}
\hline Strain or plasmid & Genotype or construction & Source or reference \\
\hline \multicolumn{3}{|l|}{ E. coli } \\
\hline CC118 $\lambda$ pir & $\Delta$ (ara-leu) araD $\triangle$ lacX74 galE galK phoA20 thi-1 rpsE rpoB argE(Am) recA1 $\lambda$ pir phage lysogen & [64] \\
\hline \multicolumn{3}{|l|}{ P. putida } \\
\hline PaW85 & Wild-type, isogenic to KT2440 & [26] \\
\hline PaWcolR & PaW85 colR::Km ${ }^{r}$ & [22] \\
\hline PaWoprB1 & PaW85 oprB1::Sm ${ }^{r}$ & [23] \\
\hline PaWcolR-oprB1 & PaWcolR oprB1::Sm ${ }^{r}$ & [23] \\
\hline PaWoprB1-tacB1 & PaWoprB1 + oprB1 under the control of tac promoter and lacla repressor $\left(\mathrm{Sm}^{r} \mathrm{Gm}^{r}\right)$ & This study \\
\hline PaWcolR-oprB1-tacB1 & PaWcolR-oprB1 + oprB1 under the control of tac promoter and lacl $\left.\right|^{q}$ repressor $\left(\mathrm{Sm}^{r} \mathrm{Gm}^{r}\right)$ & This study \\
\hline PaWcrc & PaW85 crc:: Tet ${ }^{r}$ & This study \\
\hline PaWoprB1-tacB1-crc & PaWoprB1-tacB1 crc::Tet $\left(\mathrm{Sm}^{r} \mathrm{Gm}^{r}\right.$ Tetr') & This study \\
\hline \multicolumn{3}{|l|}{ Plasmids } \\
\hline mTn5SSgusA40 & Delivery plasmid for mini $\operatorname{Tn} 5 \mathrm{Sm}\left(\mathrm{Ap}^{\mathrm{r}} \mathrm{Sm}^{r}\right)$ & [65] \\
\hline pRK2013 & Helper plasmid for conjugal transfer of mTn5SSgusA40 (Km') & [66] \\
\hline pKTlacZS/C & Promoter probe plasmid pKTlacZ containing tnpA promoter of Tn4652 fused with lacZ & [35] \\
\hline p9Tा $\mathrm{B}$ lacZ & Promoter probe plasmid $\left(\mathrm{Cm}^{r} \mathrm{Ap}^{r}\right)$ & [23] \\
\hline p9T11015 & p9TT $T_{B}$ lacZ containing gtsA promoter fused with lacZ $\left(\mathrm{Cm}^{r} \mathrm{Ap}^{r}\right)$ & This study \\
\hline pBRlacltac & Expression vector containing $P_{\text {tac }}$ promoter and lac $1^{q}$ repressor in pBR322 $\left(\mathrm{Ap}^{r}\right)$ & [67] \\
\hline pBRlacltac/oprB1 & pBRlacltac containing oprB1 as a HindllI-Xbal fragment under the $P_{\text {tac }}$ promoter $\left(\mathrm{Ap}^{r}\right)$ & This study \\
\hline pUCNotKm & pUC18Not derivative with $\mathrm{Km}^{r}$ gene instead of $\mathrm{Ap}^{r}\left(\mathrm{Km}^{r}\right)$ & R. Teras \\
\hline pUCNotKm/tacoprB1 & pUC18NotKm containing BamHI fragment with lacla-P tac-oprB1 cassette $\left(\mathrm{Km}^{\mathrm{r}}\right)$ & This study \\
\hline pBK-miniTn7- $\Omega \mathrm{Gm}$ & pUC19-based delivery plasmid for miniTn7- $\Omega \mathrm{Gm}\left(\mathrm{Ap}^{r} \mathrm{Gm}^{r}\right)$ & [68] \\
\hline pminiTn7Gm/tacoprB1 & pBK-miniTn7- $\Omega \mathrm{Gm}$ containing Notl fragment with lacl ${ }^{9}-\mathrm{P}_{\mathrm{tac}}{ }^{-o p r B 1}$ cassette $\left(\mathrm{Ap}^{\mathrm{r}} \mathrm{Gm}^{\mathrm{r}}\right)$ & This study \\
\hline pCRC10 & pKNG101 containing $s u C B$ and $c r c$ interrupted with tetracycline resistance gene $\left(\mathrm{Sm}^{r} \mathrm{Tet}^{r}\right)$ & [32] \\
\hline
\end{tabular}

incubated at $30^{\circ} \mathrm{C}$ and $E$. coli at $37^{\circ} \mathrm{C}$. Bacteria were electrotransformed following Sharma and Schimke [30].

\section{Selection of the suppressors of the lysis of the colR- deficient $P$. putida}

For the identification of genes implicated in cell lysis, the colR-deficient strain was subjected to mutagenesis using a Tn5 based mini-transposon that contains a streptomycin resistance marker. Mini-transposon-carrying plasmid mTn5SSgusA40 was conjugatively transferred from E. coli CC118 $\lambda$ pir into P. putida colRdeficient strain with the aid of the helper plasmid pRK2013. Transconjugants with random chromosomal insertions of the mini-transposon were selected on $0.2 \%$ glucose minimal plates supplemented with kanamycin, streptomycin, Congo Red and $1 \mathrm{mM}$ phenol. We searched for white colonies amongst the pink ones. Screening of about 28,000 transposon insertion derivatives of the colR-deficient strain disclosed 25 clones with significantly reduced Congo Red staining. To identify chromosomal loci interrupted in these clones, arbitrary PCR and sequencing were used. PCR products were generated by two rounds of amplification as described elsewhere [31]. In the first round, a primer specific for the Sm gene (Smsaba - 5'-GAAGTAATCGCAACATCCGC-3') and an arbitrary primer (Arb6 - 5'-GGC CACGCGTCGACTAGTACNNNNNNNNNNACGCC3') were used. Second-round PCR was performed with the primers SmSplopp (5'-GCTGATCCGGTGGAT GACCT-3') and Arb2 (5'-GGCCACGCGTCGACTAG TAC-3').

\section{Cloning procedures and the construction of bacterial strains}

For the overexpression of OprB1 in the oprB1 and colRoprB1 strains, the PCR-amplified oprB1 gene was first cloned under the control of the tac promoter and $l a c I^{q}$ repressor in pBRlacItac. oprB1 was amplified from $P$. putida PaW85 genome using oligonucleotides oprB1ees (5'-GGCAAGCTTCAAAGGCCGTTGACTCG) and oprB1lopp (5'-TGGTCTAGAGCTCTTGTTGTTTGAGAT) complementary to the upstream and downstream regions of the oprB1 gene, respectively. PCR product was cleaved with HindIII and XbaI and inserted into pBRlacItac opened with the same restrictases. The $\operatorname{lacl}^{\mathrm{q}}$ - 
$\mathrm{P}_{\text {tac }}$-oprB1 cassette was excised from pBRlacItac/oprB1 with BamHI and subcloned into BamHI-opened pUCNotKm resulting in pUCNotKm/tacoprB1. Finally, the oprB1 expression cassette was inserted as a NotI fragment into the gentamicin resistance-encoding minitransposon in the delivery vector $\mathrm{pBK}-\operatorname{miniTn} 7-\Omega \mathrm{Gm}$ yielding pminiTn7Gm/tacoprB1. To introduce the oprB1 expression cassette into the chromosome of $P$. putida PaWoprB1 or PaWcolR-oprB1, we performed triparental mating between $P$. putida strain, E. coli CC118 $\lambda$ pir carrying pminiTn7Gm/tacoprB1, and a helper plasmid pRK2013-containing E. coli HB101. Transconjugants were selected on minimal plates that contained gentamicin and streptomycin. The chromosomal presence of the lacI- $\mathrm{P}_{\text {tac }}$-oprB1 cassette of transconjugants was verified by PCR and inducible expression of OprB1 was proved by the OM protein analysis.

To disrupt the $\operatorname{crc}$ gene, the plasmid pCRC10 was employed [32]. By using triparental mating this plasmid was transferred into P. putida wild-type strain PaW85 as well as into OprB1 over-expression strain PaWoprB1tacB1. Transconjugants were first selected on tetracycline and streptomycin-containing benzoate minimal plates. Secondary screen was performed on LB plates supplemented with $10 \%$ sucrose. Sucrose-resistant colonies were picked up and the disruption of $\operatorname{crc}$ was verified by PCR using the primers PPcrcall (5'-ATC GCTACCCGATGATCTGG) and PPcrcylem (5'-TCTT GCTATCGACGATGGCG).

To make the transcriptional fusion of gtsA (PP1015) with lac $Z$ reporter gene, we used the promoter probe plasmid p9TT ${ }_{\mathrm{B}}$ lacZ. The 980-bp-long gts $A$ promoter region was amplified from $P$. putida PaW85 chromosome using oligonucleotides PP1014kesk (5'-GC TGTCGACGCCAATACGCT) and PP1015alg (5'-GCA TCTAGACGAAGCGTGGAATTCATC). The PCRamplified DNA fragment was cleaved with HincII and $\mathrm{XbaI}$ and ligated into SmaI-XbaI-opened $\mathrm{p} 9 \mathrm{TT}_{\mathrm{B}}$ lacZ, yielding p9TT1015.

\section{$\beta$-galactosidase assay}

$\beta$-galactosidase activities were measured either from solid or liquid medium-grown bacteria. For the analysis of gts $A$ promoter, total enzyme activity was measured using permeabilized cells as described elsewhere [33].

\section{Cell lysis assay}

To evaluate the cell lysis of the colR mutant, we have previously used so-called unmasked $\beta$-galactosidase assay which relies on the detection of a cytoplasmic enzyme $\beta$-galactosidase leaked out from the cells $[25,34]$. In this assay we measured the $\beta$-galactosidase activity in suspension of cells permeabilized with SDS and chloroform (total activity), and also in intact, non- permeabilized cells. The percentage of unmasked $\beta$ galactosidase activity was calculated from equation: $\mathrm{xn} /$ $\mathrm{xp} \times 100 \%$, where $\mathrm{xp}$ is $\beta$-galactosidase activity measured in SDS and chloroform-treated cells, and $\mathrm{xn}$ is $\beta$ galactosidase activity measured in non-permeabilized cells. We have shown earlier that in case of ColR-deficiency-dependent cell lysis, unmasked $\beta$-galactosidase values are above $5 \%$ [25]. As a source of $\beta$-galactosidase, the plasmid pKTlacZS/C containing the lac Z gene, was used [35]. Bacteria were grown for 24, 48, or 72 hours on glucose $(0.2,0.4$, or $0.8 \%)$ or gluconate $(0.2 \%)$ M9 minimal media. To enhance lysis, $1 \mathrm{mM}$ phenol was added to the growth medium in some experiments. Bacteria were scraped off the agar plate using plastic swabs and suspended in M9 solution. Optical density of the cell suspension was determined at $580 \mathrm{~nm}$ and $\beta$-galactosidase activity was measured [34].

\section{Isolation of outer membrane proteins}

For the isolation of outer membrane proteins (OMPs) bacteria were grown for 24 hours on two Petri plates. Bacteria were scraped off the agar and suspended in 3 $\mathrm{ml}$ of $10 \mathrm{mM}$ HEPES buffer (pH 7.4). For the analyses of peripheral and central subpopulations, bacteria were grown on agar plate in sectors as pictured in Results. To collect enough cells from the sectors, five to ten plates were used, i.e., cells from 15 to 30 sectors per strain were collected and suspended in $3 \mathrm{ml}$ of $10 \mathrm{mM}$ HEPES buffer ( $\mathrm{pH}$ 7.4). Cells were disrupted by ultrasonication and the cell debris was pelleted by centrifugation at $10000 \mathrm{~g}$ at $4^{\circ} \mathrm{C}$ for 10 minutes. The supernatant was then centrifuged at $100000 \mathrm{~g}$ at $4^{\circ} \mathrm{C}$ for 1 hour to pellet membrane proteins. In order to dissolve inner membrane proteins the pellet was resuspended in $2 \mathrm{ml}$ of $10 \mathrm{mM}$ HEPES buffer containing $1 \%$ of N-lauroylsarcosine (sodium salt) and incubated at $37^{\circ} \mathrm{C}$ for $30 \mathrm{~min}$ utes. Samples were then centrifuged at $100000 \mathrm{~g}$ at $4^{\circ} \mathrm{C}$ for 1 hour and the pellet containing OMPs was washed with $3 \mathrm{ml}$ of $10 \mathrm{mM}$ HEPES buffer. After final centrifugation at $100000 \mathrm{~g}$ at $4^{\circ} \mathrm{C}$ for 1 hour the pellet was suspended in $100 \mu \mathrm{l}$ of $10 \mathrm{mM}$ HEPES buffer. Protein concentration was measured using the Bradford assay. Two to four independent OMP preparations were made from each strain grown in particular conditions.

\section{Identification of OprE by LC-MS/MS analysis}

OM proteins were resolved by SDS-PAGE and visualized by Coomassie Blue staining. The band of interest was excised from the gel and in-gel digested with modified sequencing grade trypsin (Promega), as in [36]. Peptides from in-gel-digested samples were purified with StageTips [37] and analyzed by LC-MS/MS using an Agilent 1200 series nanoflow system (Agilent Technologies, Santa Clara, CA) connected to a LTQ Orbitrap 
classic mass spectrometer (Thermo Electron, Bremen, Germany) that was equipped with a nanoelectrospray ion source (Proxeon, Odense, Denmark). Up to five data-dependent MS/MS spectra were acquired in centroid in the linear ion trap for each FTMS full-scan spectrum. Fragment MS/MS spectra from raw files were extracted as MSM files and then merged to peak lists by using Raw2MSM version 1.7 [38] selecting the top six peaks for $100 \mathrm{Da}$. MSM files were searched with the Mascot 2.3 search engine (Matrix Science, London, UK) against the protein sequence data base composed of Pseudomonas putida KT2440 sequences and common contaminant proteins such as trypsin, keratins, etc.

\section{Measurement of residual glucose concentration in agar medium}

Bacteria were grown in three distantly located sectors on minimal agar medium containing $0.2,0.4$ or $0.8 \%$ glucose. After 24, 48, and 72 hours of growth residual glucose concentration in the agar was determined. Using sterile 1-ml pipette tips, small plugs were cut from two regions of the agar plate - just adjacent to the growth area of bacteria and underneath the cells. To excise a plug from underneath the growth area, the cells were first scraped off. Agar plugs were melted at $100^{\circ} \mathrm{C}$ and cooled to $65^{\circ} \mathrm{C}$. Glucose content in melted agar was determined with Glucose Liquicolor kit (Human GmbH, Germany) according to the instructions of the manufacturer.

\section{Results}

Glucose-specific lysis of the colR mutant occurs only on solid medium and increases in time

To specify the requirements for the glucose-related lysis of the colR-deficient P. putida, cell lysis was measured at different time points of growth both on solid and in liquid media with either glucose or gluconate as a carbon source. Cell lysis was evaluated in previously described assay [25] that measures cytoplasmic $\beta$-galactosidase leaked out from the cells (unmasked $\beta$-galactosidase activity, see Methods). Absence of ColR resulted in cell lysis only on glucose-containing solid medium and not in the liquid one (Figure 1). We observed an increase of cell lysis of the colR mutant in time until 48 hours of growth, and thereafter the unmasked $\beta$-galactosidase activity stabilized at the level of about $12 \%$ of total $\beta$-galactosidase activity (Figure 1 ). In good accordance with our previous results, colR-dependent lysis did not occur on gluconate medium [25]. These data suggest that ColRS system is particularly important for $P$. putida that grows on glucose solid medium.

\section{Identification of suppressor mutations of glucose-specific} lysis of the colR-deficient bacteria

In order to identify the genes involved in the glucosedependent cell lysis, the colR-deficient strain was

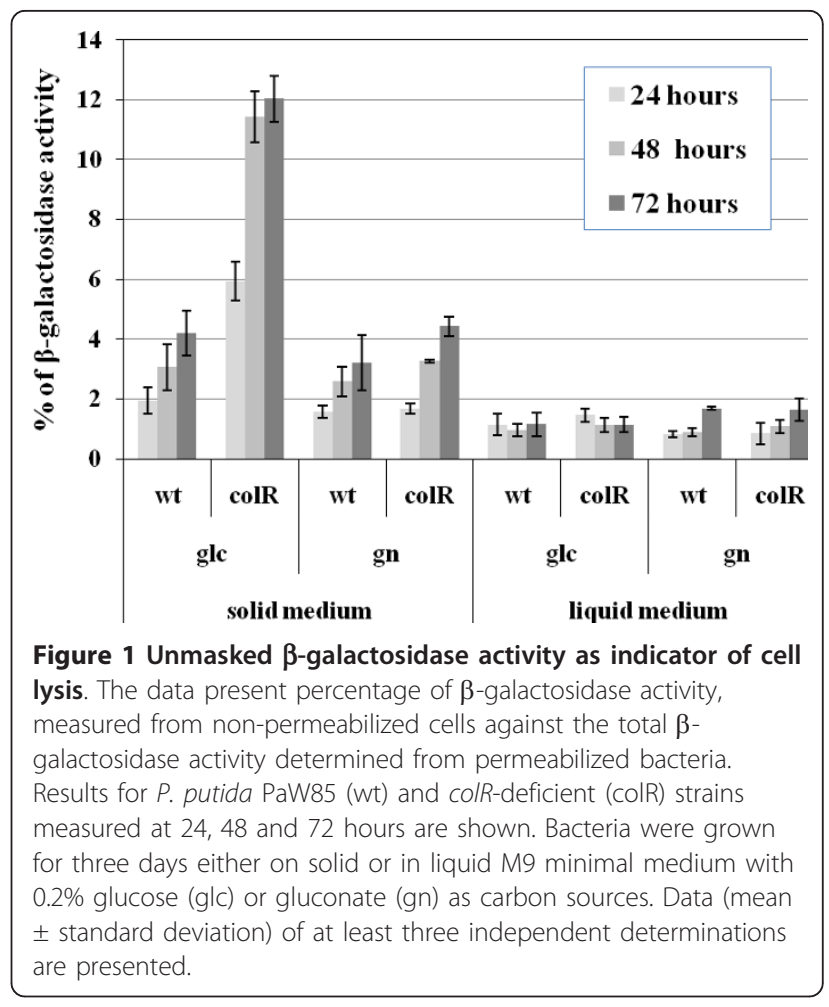

subjected to transposon mutagenesis to isolate suppressor mutations. In this screen the ability of the colR mutant colonies to bind Congo Red was used as a marker for lysis phenotype [25]: white transconjugants were searched among pinkish colR mutant colonies. As cell lysis and Congo Red binding of colR-deficient bacteria are significantly enhanced in the presence of phenol [25], suppressor screen was performed on glucose minimal plates supplemented with Congo Red and $1 \mathrm{mM}$ phenol. Analysis of about 28,000 transposon insertion derivatives of the colR-deficient strain disclosed 25 clones with significantly reduced Congo Red staining. Sequencing of mini-transposon insertions revealed 12 different suppressor genes, and most of these were picked up more than once (Table 2). The isolated white clones were tested in respect to cell lysis by using unmasked $\beta$-galactosidase assay. Data in Figure 2 show that all isolated clones not binding Congo Red also had significantly lower unmasked $\beta$-galactosidase activity compared to the parental colR-deficient strain, and most of them behaved exactly like the wild type. Thus, the results of $\beta$-galactosidase assay show a clear correlation between Congo Red binding ability and cell lysis confirming that the identified genes are indeed implicated in the glucose-specific lysis of the colR mutant.

Inspection of identified genes (Table 2) revealed that in accordance with our previous results [25], disruption of the oprB1 (PP1019) gene did eliminate the lysis. 
Table 2 List of transposon insertion derivatives of the colR mutant with reduced Congo Red staining

\begin{tabular}{|c|c|c|c|c|}
\hline Locus ID & Gene & Product name & $\begin{array}{l}\text { Number of clones } \\
\text { (disruption site)* }\end{array}$ & Probable localization\# \\
\hline PP1015 & gtsA & sugar $A B C$ transporter, periplasmic sugar binding protein & $\begin{array}{c}2 \\
(269 ; 587)\end{array}$ & $\mathrm{P}$ \\
\hline PP1016 & gts $B$ & sugar $A B C$ transporter, permease protein & $\begin{array}{c}2 \\
(717 ; 972)\end{array}$ & CM \\
\hline PP1018 & gts $D$ & sugar $A B C$ transporter, ATP-binding protein & $\begin{array}{c}2 \\
(414 ; 1094)\end{array}$ & CM \\
\hline PP1019 & oprB1 & porin B1 & $\begin{array}{c}3 \\
(66 ; 146 ; 909)\end{array}$ & $\mathrm{OM}$ \\
\hline PP1345 & $\sec A$ & preprotein translocase subunit SecA & $\begin{array}{c}2 \\
(2 \times 2695)\end{array}$ & C \\
\hline PP1585 & & antidote protein, putative & $\begin{array}{c}1 \\
(203)\end{array}$ & unknown \\
\hline PP2088 & $\operatorname{sig} x$ & extracytoplasmic function sigma factor SigX & $\begin{array}{c}4 \\
(251 ; 304 ; 336 ; 480)\end{array}$ & C \\
\hline PP2089 & oprF & porin $F$ & $\begin{array}{c}1 \\
(849)\end{array}$ & $\mathrm{OM}$ \\
\hline PP4236 & $d s b E$ & thiol:disulfide interchange protein DsbE & $\begin{array}{c}1 \\
(526)\end{array}$ & CM \\
\hline PP4695 & cbrA & sensor histidine kinase CbrA & $\begin{array}{c}4 \\
(2 \times 10 ; 936 ; 1763)\end{array}$ & CM \\
\hline PP4696 & $c b r B$ & response regulator $\mathrm{CbrB}$ & $\begin{array}{c}1 \\
(1013)\end{array}$ & C \\
\hline PP5053 & $\sec B$ & preprotein translocase subunit SecB & $\begin{array}{c}2 \\
(9 ; 314)\end{array}$ & C \\
\hline
\end{tabular}

* The numbers in the brackets denote nucleotides in the coding sequence of particular gene just before the minitransposon insertion.

\# Abbreviations: CM - cytoplasmic membrane, OM - outer membrane, C - cytoplasm, P - periplasm

Knockouts of sugar transport genes located upstream of oprB1, i.e., gtsA (PP1015), gtsB (PP1016), and gtsD (PP1018) also suppressed the lysis phenotype of the colR mutant. In addition to sugar transport genes, lysis was also suppressed by inactivation of the two-component system CbrA-CbrB, which is known to regulate several catabolic pathways and the cellular ratio of carbon to nitrogen $[39,40]$. The death of the colR mutant was also prevented by the knockout of a sigma factor SigX, which regulates expression of major outer membrane protein OprF in Pseudomonas aeruginosa and Pseudomonas fluorescens [41]. Consistent with that, inactivation of $o p r F$ also suppressed lysis of the colR mutant. It is noteworthy that the disruption of the SecA and SecB components of the general Sec protein secretion pathway also eliminated the lysis (Table 2). The isolation of a $\sec A$-knockout in our screen was particularly surprising because SecA has been shown essential not only for Sec pathway but also for the viability of bacteria [42]. Sequencing of two independently identified $\sec A$ mutants revealed that they both possessed minitransposon insertion at the very end of the $\sec A$ gene - between 37 and 38 nt from the stop codon (Table 2). Therefore, these mutants most probably coded for a truncated SecA protein lacking the last 12-13 amino acids. The most distal C-terminal part of SecA is necessary for its binding with $\operatorname{SecB}$, a chaperone which keeps precursor proteins in translocation-competent state and targets them to SecA [42]. Literature data shows that although SecA is essential for bacteria, its SecB-binding domain is dispensable for protein secretion and cell viability $[43,44]$. Thus, we consider that the $\sec A$ mutants that were picked up in our suppressor screen are impaired only in SecB-dependent protein secretion and in respect of the cell lysis phenotype they resemble $\sec B$-knockouts. Finally, unique insertions of transposon into PP1585 and PP4236, coding for putative antidote protein of a toxin-antitoxin system and a thiol:disulfide interchange protein, respectively, also resulted in white non-lysing colonies of the colR mutant. In conclusion, inactivation of different genes prevented lysis of the colR mutant and most of these genes encode either membrane proteins or are implicated in regulating membrane proteins.

Analysis of the outer membrane composition of the nonlysing transposon derivatives of the colR mutant

The results of the suppressor analysis predict that the colR mutant cannot maintain membrane protein homeostasis. This is supported by two phenomena. First, the 


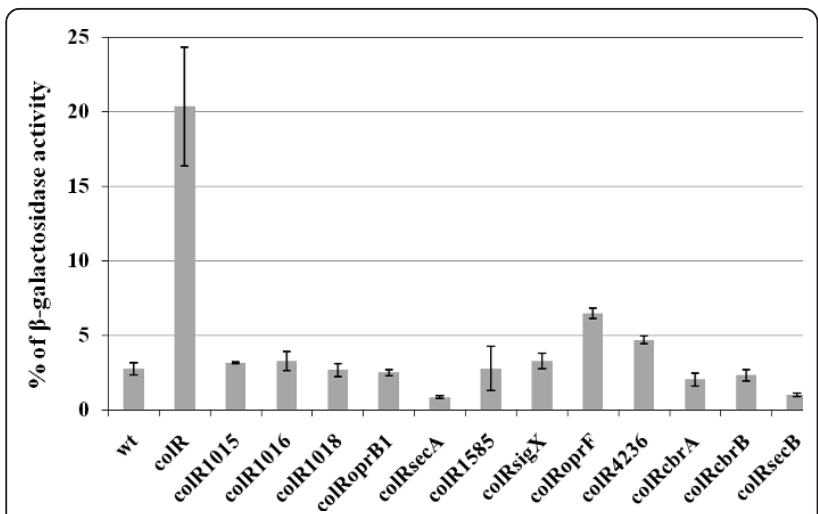

Figure 2 Unmasked $\beta$-galactosidase activity as indicator of cell lysis of Congo Red non-binding derivatives of the colRdeficient strain. The data present percentage of $\beta$-galactosidase activity, measured from non-permeabilized cells against the total $\beta$ galactosidase activity determined from permeabilized bacteria. Results for P. putida PaW85 (wt), colR-deficient strain (colR), and for different transposon insertion derivatives of the colR mutant are shown. Bacteria were grown for 24 hours on solid $0.2 \%$ glucose M9 minimal medium containing $1 \mathrm{mM}$ phenol. Data (mean \pm standard deviation) of at least three independent determinations are presented.

reduction of protein secretion by the inactivation of the SecB-dependent protein export suppresses cell lysis. Second, the disruption of genes for the outer membrane porins, OprB1 and OprF, also eliminated the lysis indicating that the outer membrane (OM) composition may be unbalanced in the colR-deficient $P$. putida. In order to address this issue we compared the pattern of OM proteins of the wild-type and the colR mutant as well as the suppression mutants of the colR strain. Data in Figure 3 demonstrate that the overall OM protein pattern of the wild-type and the colR strains is similar. The PP1585, PP4236, secA and secB derivatives of the colR mutant also have OM protein profiles that are quite similar to the wild-type. However, as expected, OM protein preparations of the colRoprB1 and colRoprF mutants respectively lacked OprB1 and OprF channel proteins. Note that OprF is represented by several differently migrating forms. This is consistent with previous data on several OM porins, including OprF of $P$. aeruginosa, showing that these proteins are prone to modification by heat and $\beta$-mercaptoethanol treatment that is carried out for the solubilization of proteins before applying to the gel [45]. Given that $\operatorname{sig} X$ and oprF genes comprise one operon and that OprF is positively regulated by SigX in $P$. aeruginosa and $P$. fluorescens [41], it was expected that all four different colRsigX knockout strains have significantly lowered OprF amount in their OM (Figure 3, only two colRsigX derivatives are presented). However, while three $\operatorname{sig} X$ derivatives of the colR mutant (minitransposon insertions after nucleotides 251, 304 and 336 of the $\operatorname{sig} X$ gene) revealed only modestly reduced expression of OprF (Figure 3, only colRsigX $X_{336}$ is presented), the colRsigX strain with most distal transposon insertion in $\operatorname{sig} X$, displayed drastically decreased OprF level (Figure 3, see colRsig $X_{480}$ ). An analysis of the sugar transport gene knockouts (PP1015, PP1016 and PP1018) showed that they all possessed a significantly decreased amount of OprB1. Thus, disruption of the genes located upstream of oprB1 seems to have a polar effect on the OprB1 expression. Actually, this is in good agreement with recent results reporting that sugar transport genes comprise one transcriptional unit with oprB1 [46]. OM fractions of colRcbrA and colRcbrB mutants were generally similar to the wild-type and the colR mutant, but still had slightly less OprB1 protein than the parental strain. Thus, OM analysis shows that although the pattern of OM proteins of the colR mutant resembles that of the wild-type, its

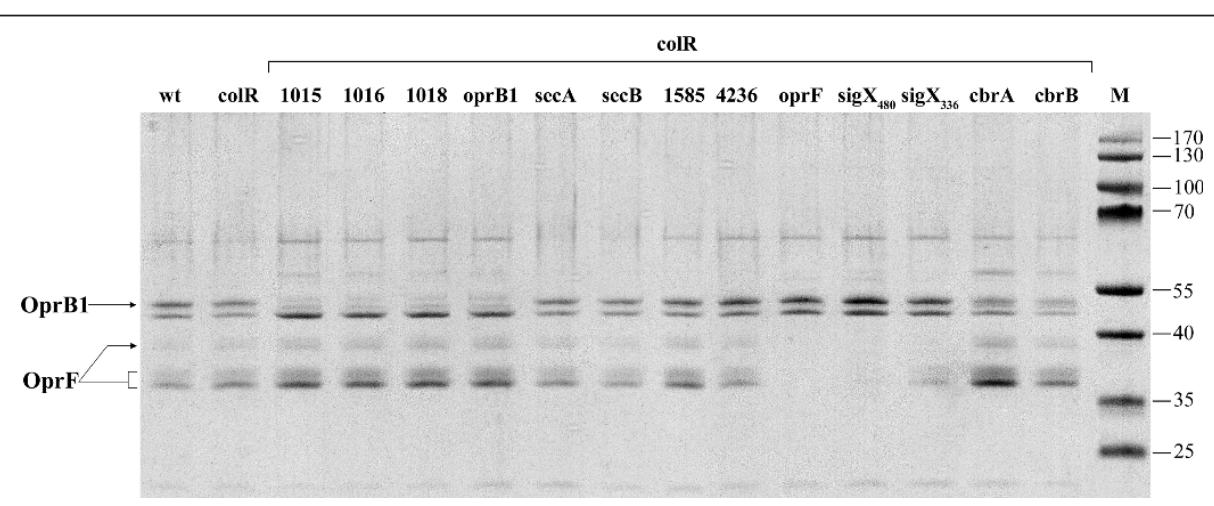

Figure 3 SDS-PAGE of outer membrane protein preparations stained with Coomassie Blue. OM proteins were extracted from 24-hour-old populations of bacteria grown on solid minimal medium with $0.2 \%$ glucose. Representative results of the P. putida PaW 85 (wt), colR-deficient (colR), and of different transposon insertion derivatives of the colR-deficient strains are shown. Arrows indicate locations of the channel proteins OprB1 and OprF (calculated molecular weights $49.6 \mathrm{kD}$ and $37 \mathrm{kD}$, respectively). All lanes contain $0.5 \mathrm{\mu g}$ of OM proteins. 
defects can be suppressed by decreasing the amount of OprB1 or OprF in OM.

\section{Overexpression of OprB1 induces cell lysis, especially in the colR-deficient background}

The analysis of the OM protein pattern of transposon mutants suggested that the colR-deficient $P$. putida cannot tolerate the natural load of membrane proteins, at least that of OprB1 and OprF when growing on glucose solid medium. Here, it is important to note that the colR mutant is prone to lysis specifically on glucose but not on gluconate [25] despite both these substrates are degraded through Entner-Doudoroff pathway. While most of the genes for glucose and gluconate metabolism are induced by both these carbon sources, one of them, oprB1, is specifically expressed only during glucose growth $[46,47]$. Our results also show that OprB1, a major OM protein in glucose-grown cells, is not detectable in gluconate-grown P. putida (Figure 4A). Therefore, we hypothesized that the glucose-induced expression of OprB1 could be the major determinant of glucose-specific cell lysis of the colR-deficient bacteria. If so, then artificial overexpression of OprB1 should result in the cell lysis of the colR mutant on both the glucose and the gluconate medium. To test this assumption, we introduced an extra copy of the oprB1 gene under control of IPTG-inducible tac promoter to the oprB1-deficient strains PaWoprB1 and PaWcolR-oprB1. The oprB1-deficient background was used to avoid an unequal amount of OprB1 in glucose and gluconate growing cells due to glucose-specific induction of the native oprB1 locus. The OM analysis of PaWoprB1-
tacB1 and PaWcolR-oprB1-tacB1 strains revealed that induction of tac promoter with $0.5 \mathrm{mM}$ IPTG resulted in equal OprB1 expression in both strains and in case of both carbon sources (Figure 4B). OprB1 protein was not detected in cells without IPTG-induction (not shown). Unmasked $\beta$-galactosidase activity assay demonstrated that overexpression of OprB1 caused the lysis of the colR mutant also on the gluconate medium (Figure $4 \mathrm{C}$ ), which confirms the importance of the amount of OprB1 in $\mathrm{OM}$ as a major determinant of cell lysis. Furthermore, even the colR-proficient PaWoprB1-tacB1 strain did not tolerate the artificial overexpression of OprB1, revealing a clear lysis phenotype on both carbon sources. This data suggests that $\mathrm{OM}$ is highly sensitive to the abundance of OprB1 and obviously the natural amount of OprB1 induced by glucose is close to the saturating level that the bacterium can tolerate.

\section{The degree of lysis of the colR mutant depends on the location of cells in the solid medium population and on the glucose concentration in the medium}

Two remarkable features of the glucose-specific cell lysis of the colR-deficient strain are that it can be observed only on solid medium (Figure 1) and that only a fraction of population lyses [25] indicating heterogeneity among the bacteria. Therefore we decided to test the effect of the location of cells in a population on their lysis. For that, the colR-deficient bacteria were grown on agar plates with $0.2 \%$ glucose and lysis was analysed in cells withdrawn from two different regions of bacterial lawn on agar plate sectors - the periphery and the centre. Bacteria were streaked as shown in Figure 5A to

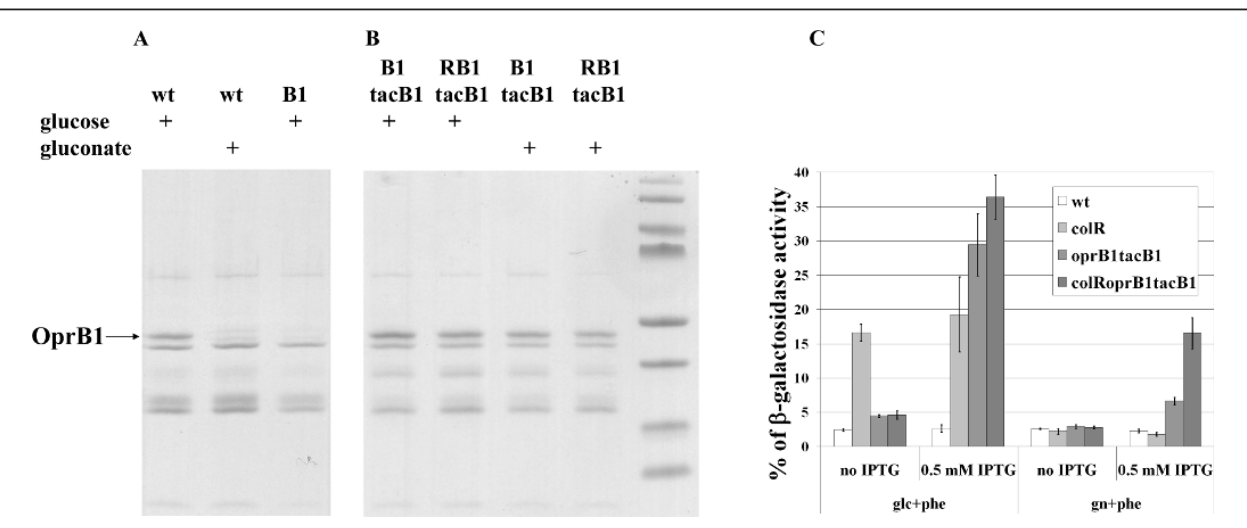

Figure 4 Effect of the OprB1 overexpression on the profile of outer membrane proteins and cell lysis. A and B. SDS-PAGE of outer membrane protein preparations stained with Coomassie Blue. Representative results of the P. putida PaW85 (wt), oprB1-deficient (B1) as well as OprB1-overexpressing strains PaWoprB1-tacB1 (B1tacB1) and PaWcolR-oprB1-tacB1 (RB1tacB1) are presented. OM proteins were extracted from 24hour-old populations of bacteria grown on solid minimal medium with either $0.2 \%$ glucose or gluconate. OM proteins presented in panel $B$ have been purified from the cells which were grown in the presence of $0.5 \mathrm{mM} \mathrm{IPTG.} \mathrm{Plus} \mathrm{(+)} \mathrm{marks} \mathrm{above} \mathrm{the} \mathrm{lanes} \mathrm{designate} \mathrm{a} \mathrm{particular}$ carbon source added to the growth medium. Arrow indicates location of OprB1. C. Quantification of cell lysis by the unmasked $\beta$-galactosidase assay. Bacteria were grown for 24 hours on solid $0.2 \%$ glucose (glc) or $0.2 \%$ gluconate (gn) minimal medium containing 1 mM phenol (+phe). For the induction of OprB1 0.5 mM IPTG was used. Data (mean \pm standard deviation) of at least three independent determinations are presented. 
A

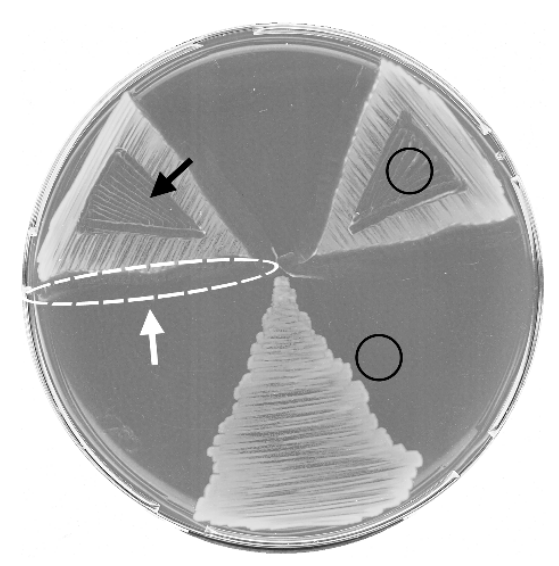

C

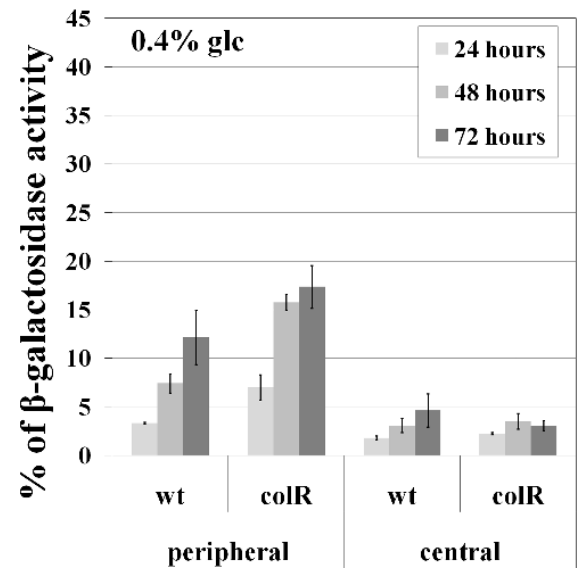

B

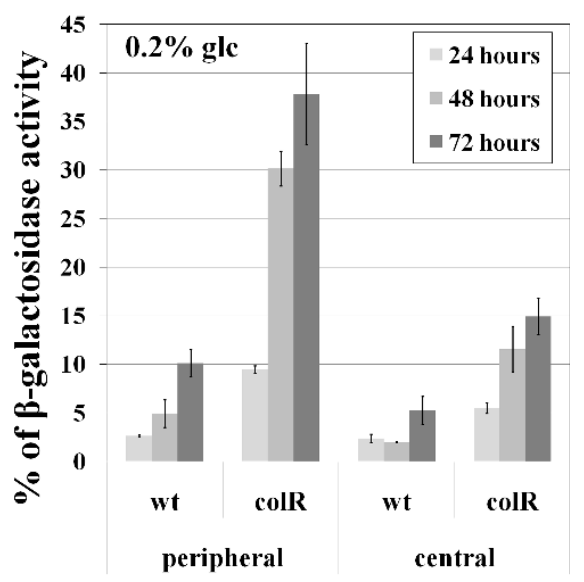

D

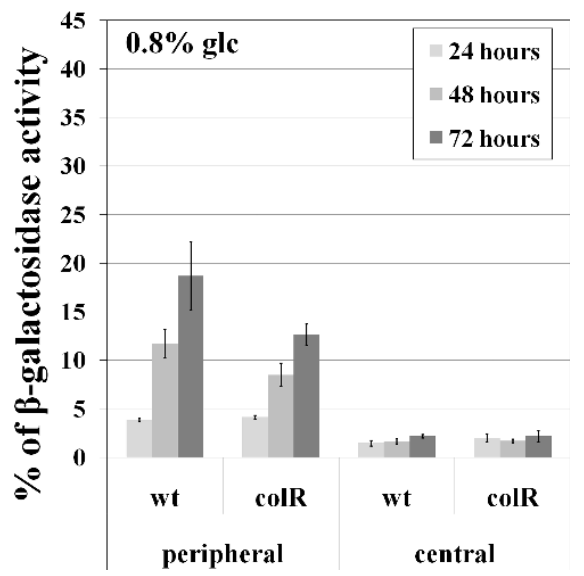

Figure 5 Comparison of lysis of peripheral and central subpopulations of $P$. putida PaW85 wild-type (wt) and colR-deficient (colR) strains grown on solid glucose medium. A. Representation of a Petri plate with three growth sectors of bacteria and subpopulations sampled for $\beta$-galactosidase analysis. Unmasked $\beta$-galactosidase activity was assayed from the cells of peripheral subpopulation (area encircled by the dotted line and indicated by the white arrow) and from central one (indicated by the black arrow). Black circles indicate the areas sampled for the measurement of residual glucose concentration in the medium (data is presented in Table 3). The degree of lysis is presented as unmasked $\beta$-galactosidase activity which was measured from bacteria grown either 24,48 or 72 hours on solid media with $0.2 \%$ (B), $0.4 \%$ (C) or $0.8 \%$ (D) of glucose (glc) as the carbon source.

enhance the build-up of nutrient gradients. Unmasked $\beta$-galactosidase activity measured at 24,48 and 72 hours of growth clearly indicated that at every time-point the lysis of colR mutant was always significantly higher among peripheral cells of the bacterial lawn compared to the central subpopulation (Figure 5B). Also wild-type bacteria revealed spatially different unmasked $\beta$-galactosidase activity demonstrating up to two-fold higher enzyme values at 48 and 72 hours in case of peripheral cells (Figure 5B).

Due to the spatiotemporal character of the lysis of the colR mutant we hypothesized that nutrient limitation could be involved in cell death. During the active growth of bacteria on agar plate the concentration of glucose in the growth area decreases, yet, it is obvious that compared to the central population the peripheral cells are nutritionally less limited due to diffusion of glucose from the adjacent medium. To evaluate the glucose consumption dynamics during 72 hours of bacterial growth on $0.2 \%(9 \mathrm{mM})$ glucose solid medium, we measured the glucose concentration in the growth agar by sampling the regions underneath the cell lawn and adjacent to the bacterial growth area (sampling regions are indicated in Figure 5A). Already at 24 hours of growth, the amount of glucose in the medium underneath the bacterial lawn had dropped below the detection level of the assay $(0.1 \mathrm{mM})$. Concentration of glucose in the medium adjacent to the growth area continuously 
dropped down to $1.6 \mathrm{mM}$ by 72 hours of growth (Table $3)$. These results show that bacteria constantly consume glucose that is diffusing from adjacent region of agar plate and that peripheral population of bacteria has to adapt to gradient of glucose. Notably, glucose consumption dynamics for the wild-type and the colR mutant were similar.

Accumulating evidence indicates that bacteria growing under subsaturating nutrient levels express a transient response called hunger response, which helps them to cope with limiting conditions [48]. The most obvious feature of hunger response is up-regulation of nutrient uptake systems, including several OM porins $[3,5]$. This lead us to hypothesize that elevated lysis of peripheral cells on $0.2 \%$ glucose plates resulted from the hunger response of bacteria while central cells were already starving at that time and therefore showed only minimal hunger response. If so, then the lysis of peripheral cells should be suppressed by increasing the glucose concentration in the medium. Thus, we assessed the cell lysis of peripheral and central subpopulations under different glucose concentrations. Data in Figure $5 \mathrm{C}$ and $5 \mathrm{D}$ clearly shows that the lysis of the colR-deficient strain inversely correlates with the glucose concentration in the medium. While the increase of the initial glucose concentration in the medium up to $0.4 \%$ (two-fold) had no effect on the unmasked $\beta$-galactosidase activity of the wild-type (compare Figure 5B and 5C), in colR-deficient background this increase significantly reduced the lysis of peripheral cells and eliminated the lysis of central cells (Figure 5C). If the growth medium of bacteria contained $0.8 \%$ of glucose instead of $0.2 \%$, then both peripheral and central subpopulations of colR mutant behaved similarly to the wild-type, i.e., showed no ColRdepletion-dependent lysis (Figure 5D). In a parallel experiment we also monitored the glucose concentration in the agar plate and observed that after 24 hours of growth the glucose was already exhausted (residual concentration below $0.1 \mathrm{mM}$ ) from underneath the cell lawn even if the initial glucose concentration in the medium was 0.4 or $0.8 \%$. At the same time, the glucose concentration in the adjacent medium was relatively high although it was constantly decreasing over time (Table 3). There was an inverse correlation between the lysis of peripheral cells of colR-mutant and glucose

Table 3 Glucose concentration in the bacteria-free agar medium adjacent to the growth area of the cells

\begin{tabular}{llll}
\hline \multicolumn{4}{l}{ Glucose concentration $(\mathrm{mM})$} \\
\hline Initially & After $\mathbf{2 4}$ hours & After $\mathbf{4 8}$ hours & After $\mathbf{7 2}$ hours \\
\hline $9(0.2 \%)$ & $6.9 \pm 0.3$ & $2.9 \pm 0.6$ & $1.6 \pm 0.2$ \\
\hline $18(0.4 \%)$ & $14.0 \pm 1.0$ & $5.9 \pm 0.4$ & $3.5 \pm 0.4$ \\
\hline $36(0.8 \%)$ & $29.2 \pm 0.3$ & $13.0 \pm 1.3$ & $6.8 \pm 0.9$ \\
\hline
\end{tabular}

concentration adjacent to the growth area - irrespective of the initial glucose concentration $(0.2,0.4$, or $0.8 \%)$, the lower the glucose concentration in adjacent region was, the greater was the lysis (Table 3 and Figure 5). If initial glucose concentration in the medium was $0.8 \%$, it did not decrease below $6 \mathrm{mM}$ in the region adjacent to the cell growth area during the experiment (Table 3). This level is obviously too high to initiate the lysis of the colR-deficient strain. This data strongly suggests that particularly the hungry fraction of the colR mutant is liable to lysis.

\section{Amount of OprB1 in OM inversely depends on glucose concentration}

After establishing conditions which enhance (peripheral growth) and diminish (higher glucose concentration) the lysis of colR mutant cells, we asked whether we can see some changes in the OMP composition under respective conditions. As the abundance of OprB1 in OM was promoting cell lysis, we hypothesised that the level of OprB1 may inversely depend on glucose concentration. To test that, we analysed the pattern of OM proteins of the wild-type and the colRdeficient bacteria grown on agar plates with different concentrations of glucose. Analysis of OMP fraction purified from peripheral subpopulations revealed a dissimilar OM protein pattern for bacteria grown at different glucose concentrations (Figure 6A). First, the OM preparations of bacteria grown at 0.4 or $0.8 \%$ of glucose revealed an additional OM protein ( 50 kD) that was barely detectable in the membrane preparations of bacteria grown at $0.2 \%$ of glucose. A similar pattern was observed also for the OMP preparation of central cells (data not shown). Mass spectrometric analysis identified this hunger-repressed protein as OprE encoded by PP0234 (Figure 6A). Second, the amount of OprB1 inversely correlated with initial glucose concentration in agar plates being highest at $0.2 \%$ and lowest at $0.8 \%$ of glucose (Figure $6 \mathrm{~A}$ ). Note that the differences observed for OprB1 amounts in OM correlated well with the lysis data of the colR mutant on different glucose plates (Figure 5). All these results support the hypothesis that an elevated expression of OprB1 due to nutrient limitation generates membrane stress that is not tolerated by the colR mutant and results in the lysis of most vulnerable subpopulation of bacteria.

When analysing the composition of OM proteins of bacteria grown on $0.2 \%$ glucose (conditions that promote lysis), we repeatedly observed a slight difference between the wild-type and the colR mutant regarding the relative proportions of OprB1 and OprF. The colR mutant showed a tendency to have less OprB1 and more OprF in OM than the wild-type. This was most 

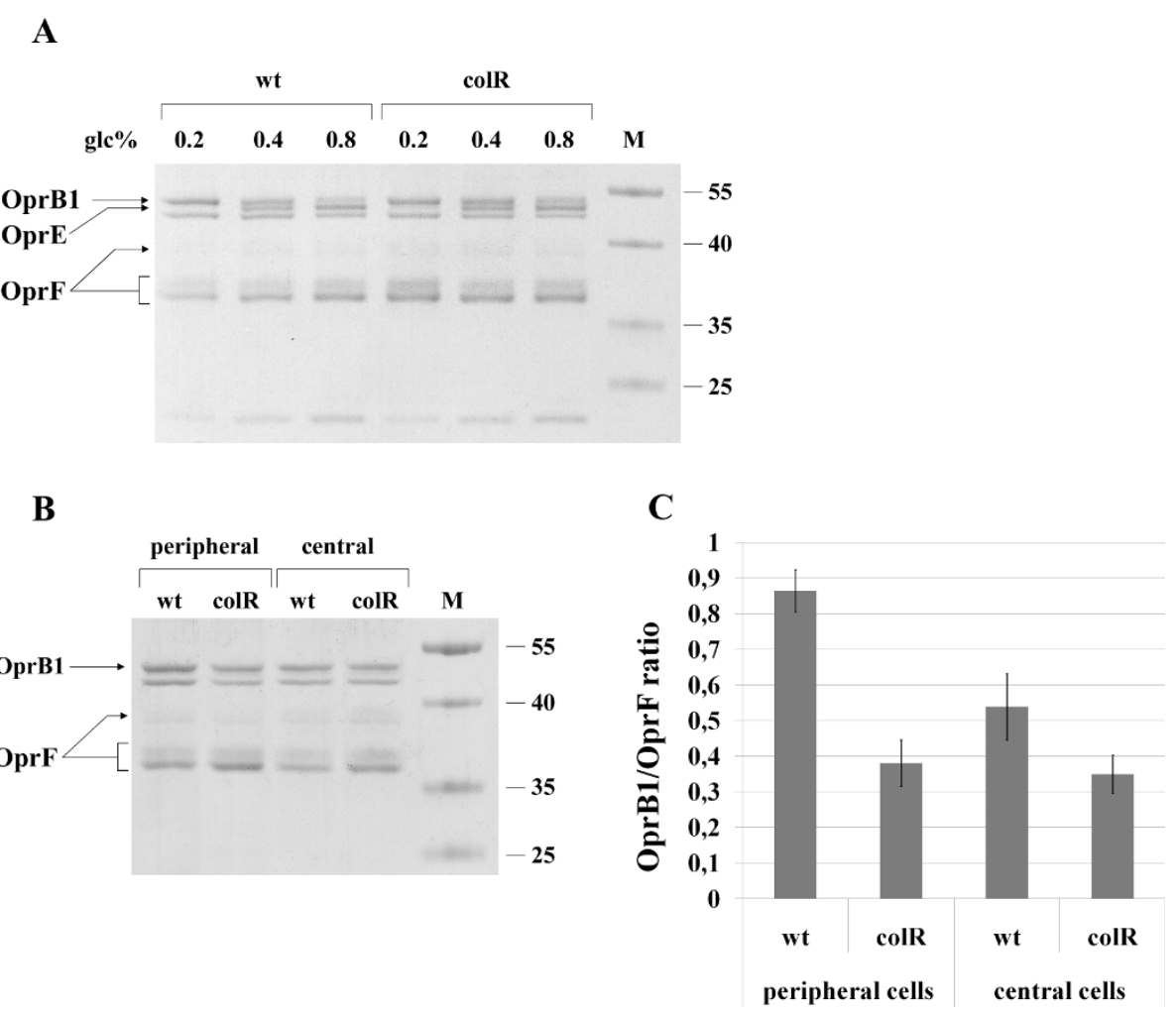

Figure 6 Profiles of the outer membrane proteins of the P. putida PaW85 (wt) and the colR-deficient (colR) strains under different growth conditions. OM proteins were purified from the solid medium-grown P. putida PaW85 (wt) and colR-deficient (colR) strains cultivated on the agar plate sectors as illustrated in Figure 5A. A. OM protein profiles of 24-hour-old peripheral subpopulations of bacteria grown on solid medium with $0.2,0.4$ or $0.8 \%$ glucose. Location of OprB1, OprE, and OprF is indicated by the arrows. B. OM protein profiles of peripheral and central subpopulations grown for 24 hours on $0.2 \%$ glucose solid medium. The quantified protein bands are indicated by the arrows. C. The ratio of OprB1 to OprF in different subpopulations of the $P$. putida wild-type and the colR mutant strains grown for 24 hours on $0.2 \%$ glucose solid medium. The OprB1/OprF ratio was calculated from the data obtained from at least two independent protein preparations and from three independent gel runs. Mean values and 95\% confidence intervals are presented.

clearly seen when the OM protein profiles of peripheral subpopulations of two strains were compared (for representative results see Figure $6 \mathrm{~B}$ ). In order to quantify the proportions of OprB1 and OprF in the OMP preparations, we analysed the SDS-PAGE images with ImageQuant TL program. Quantification showed that OM of the wild-type indeed contained relatively more OprB1 than that of the colR-deficient strain (Figure $6 \mathrm{C}, \mathrm{p}=$ 8,6e-07 and $\mathrm{p}=6,8 \mathrm{e}-04$ for preparations from peripheral and central cells, respectively). Furthermore, the relative amount of OprB1 was higher in peripheral wild-type cells compared to that of central cells $(p=6,2 \mathrm{e}-05)$, indicating the elevated hunger response of peripheral cells. Differently from the wild-type, the OprB1/OprF ratio for the peripheral and the central cells of the colR mutant was similar. We suggest that the increased level of OprB1 in OM that is normally induced in response to glucose limitation is unbearable to the colR mutant and therefore does not rise above a certain threshold level.

\section{Hunger-induced expression of OprB1 is regulated post- transcriptionally}

To test the possibility that expression of OprB1 under glucose limitation increases due to enhanced transcription of glucose transport operon (genes gts $A$ to oprB1), the transcriptional fusion of $g t s A$ with $l a c Z$ reporter was constructed and analysed under different glucose concentrations. Results in Figure 7A show that the expression of the gtsA promoter is induced by glucose regardless of its concentration. This was also confirmed in the liquid glucose medium by $\beta$-galactosidase measurements throughout the growth (data not shown). To find out whether OprB1 expression may be regulated post-transcriptionally we employed the PaWoprB1-tacB1 and PaWcolR-oprB1-tacB1 strains with oprB1 gene under the control of IPTG-inducible tac promoter. We presumed that if the expression of OprB1 is post-transcriptionally suppressed at high glucose and, vice versa, derepressed under glucose limitation, then it should not be possible to artificially overexpress OprB1 from tac 
A

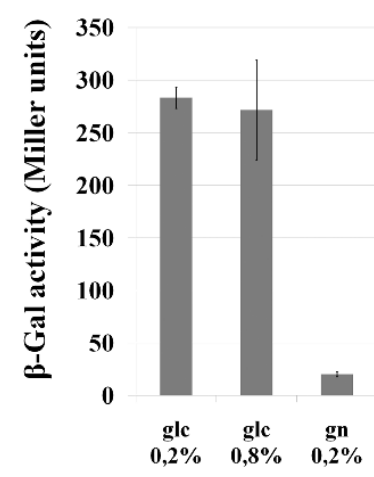

C

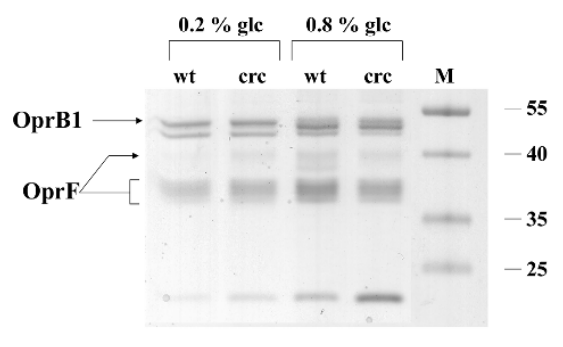

B

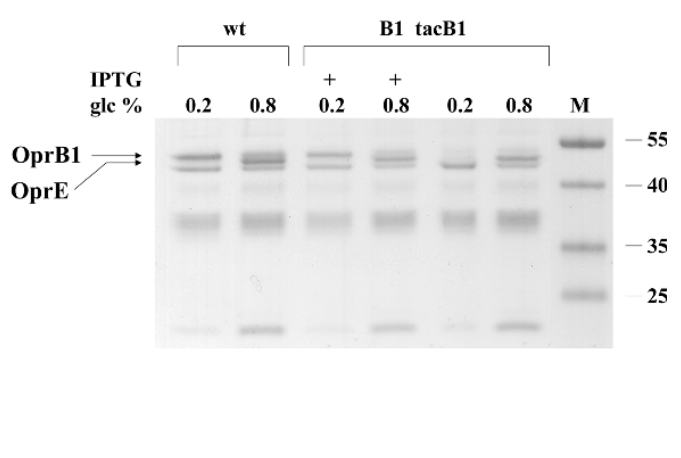

D

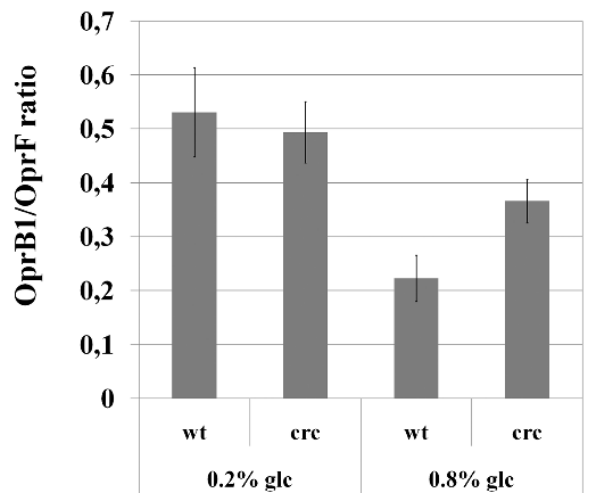

Figure 7 Post-transcriptional regulation of OprB1 depends on the glucose concentration. A. $\beta$-Galactosidase ( $\beta$-Gal) activity expressed from the gtsA promoter was measured in the wild-type $P$. putida grown on solid medium with 0.2 or $0.8 \%$ glucose or $0.2 \%$ gluconate. B. SDSPAGE of the outer membrane protein preparations from P. putida wild-type PaW85 (wt) and from OprB1-overexpressing strain PaWoprB1-tacB1 (B1tacB1) grown 24 hours over the whole Petri plate. The growth medium contained 0.2 or $0.8 \%$ glucose (glc) as a carbon source. Plus (+) mark above the lane indicates that the bacterial growth medium contained also $0.5 \mathrm{mM}$ IPTG. C and D. Analysis of the effect of the crc inactivation on the hunger-induced up-regulation of OprB1. The outer membrane proteins were prepared from P. putida wild-type (wt) and crc mutant strains (crc) grown for 24 hours as a lawn over the entire Petri plate. The growth medium contained 0.2 or $0.8 \%$ glucose (glc). The ratio of OprB1 to OprF was calculated from the data of at least two independent protein preparations and five independent gel runs. Mean values and $95 \%$ confidence intervals are presented.

promoter in glucose-rich environment, i.e., on $0.8 \%$ glucose medium. As predicted, the tac promoter-originated artificial expression of OprB1 was lower at $0.8 \%$ glucose compared to that at $0.2 \%$ glucose (Figure $7 \mathrm{~B}$ ). As a matter of fact, it did not exceed the amount of OprB1 characteristic for the wild-type cells growing on glucose-rich medium. This data strongly suggests that hunger-dependent regulation of OprB1 occurs post-transcriptionally. Here, it is relevant to remind that the amount of OprB1 is slightly reduced in $c b r A$ and $c b r B$ mutants (Figure 3) suggesting that the CbrA-CbrB system is involved in the OprB1 regulation. Recently, CbrA-CbrB system has been shown to act as a positive regulator of $\mathrm{CrcZ}$ which is an antagonist sRNA of catabolite repression control protein Crc [49]. The RNA-binding Crc is a global translational regulator of catabolite repression in pseudomonads
[50-52]. Interestingly, if $P$. putida grows on amino acidrich LB medium, the glucose transport genes are repressed by Crc [53]. Furthermore, sequences similar to $\mathrm{Crc}$ binding consensus were found in the proximity of the AUG start site of $g t s A$ and oprB1 genes [50]. The Crc protein therefore seemed to be a likely candidate for translational repression of OprB1 in the glucose-rich solid medium. Thus, we constructed the $c r c$-deficient strains and analyzed the effect of Crc inactivation on the amount of OprB1 in OM under glucose-rich (0.8\%) and glucose-limiting $(0.2 \%)$ conditions. Data in Figure 7 (panels $\mathrm{C}$ and D) show that $\mathrm{Crc}$ indeed affects the relative abundance of OprB1 in OM; yet, this was observed only under glucose-rich conditions in which $\mathrm{crc}$ mutant displayed higher OprB1/OprF ratio than the wild-type $(\mathrm{p}=8.6 \mathrm{e}-05)$. However, although inactivation of $\mathrm{crc}$ 
alleviated repression of $\mathrm{OprB1}$ on $0.8 \%$ glucose medium, the OprB1/OprF ratio was still higher on $0.2 \%$ glucose medium (Figure 7D, compare results for the crc mutant on 0.2 and $0.8 \%$ glucose, $\mathrm{p}=6.7 \mathrm{e}-04)$. Therefore we conclude that in addition to the Crc some other factor (s) as yet unknown should be implicated in hungerinduced up-regulation of OprB1.

\section{Discussion}

Previous studies on ColRS signaling system have revealed a peculiar subpopulation lysis phenotype of the colR mutant grown on glucose solid medium [25]. In this study we clarified the reasons for glucose-specific cell lysis and revealed that the ColRS system is necessary for $P$. putida to survive the hunger response which includes up-regulation of sugar channel OprB1.

Several lines of evidence obtained in this study suggest that the glucose-growing colR mutant experiences envelope stress caused by the accumulation of membrane proteins. This was first indicated by the collection of mutants suppressing the lysis phenotype of the colRdeficient strain. These data demonstrated that the loss of ColR can be suppressed by down-regulation of certain OM proteins like OprB1 and OprF, as well by hindering the SecB-dependent protein secretion. Second, artificial overexpression of sugar channel protein OprB1 further highlighted the specifically increased sensitivity of the colR mutant to this particular OM protein. Although neither the wild-type nor the colR-deficient strain tolerated the elevated expression of OprB1 exhibiting lysis phenotype on both glucose and gluconate medium, the colR mutant was remarkably more affected (Figure 4C). Third, the colR-deficient strain possessed slightly less OprB1 in its OM than the wild-type (Figure $6 \mathrm{C})$, indicating that the membrane of the colR mutant is probably sensitive to accumulation of OprB1. Thus, our data suggest that ColRS is necessary for P. putida to maintain the cell membrane homeostasis and this becomes particularly important during up-regulation of certain OMPs such as OprB1.

We detected the glucose-specific cell lysis of the colRdeficient strain only on solid and not in liquid medium (Figure 1). Bacterial population growing on solid medium is highly heterogeneous and it is obvious that bacteria located at the edge of the growth area experience different conditions compared to the cells in the centre of the population. Gradient fields of carbon source as well as of excreted metabolites develop during the growth, putting the cells in the centre of the population under more restrictive conditions than those at the periphery. It has been shown that such gradient fields govern cellular responses of multicellular solid medium populations and regulate development of gene expression patterns in space and time [11]. Our previous results revealed a spatial aspect of ColR-dependent lysis. Colonies of the colR-deficient strain developed central concavities when growing on the glucose medium which we interpreted as an elevated lysis of central population [25]. Here, we proved that the degree of lysis of the colR mutant is spatially different. However, contrary to our expectations the lysis of peripheral cells was significantly higher than that of the central cells. Yet, it is important to point out that in the current study we analyzed the bacteria grown on a sector ( $1 / 6$ of the Petri plate), the area of which is more than 100 times bigger than that of a single colony. Therefore, the nutrient gradients building up in the medium under central cells of a sector and under the central part of a colony are not really comparable. We suggest that lysis occurs at a certain glucose concentration range and whether this develops in the centre or in the periphery of a population depends on the size of the cells' growth area.

This study indicated that the glucose-specific lysis of the colR-deficient $P$. putida occurs among a subpopulation of cells adapting to nutrient limitation. This was most strongly evidenced by the fact that the degree of lysis depended both on time and glucose concentration. We suggest that the continuous increase of the colR mutant lysis during the first 48 hours of growth on $0.2 \%$ glucose solid medium (Figure 1 and Figure 5) is caused by a gradual decrease of glucose concentration. Given that significantly less lysis was observed on $0.4 \%$ glucose and that no lysis was detected on $0.8 \%$ glucose medium (Figure 5), it is possible to conclude that the ColRdependent cell lysis occurs only when the amount of glucose decreases below a certain threshold level. However, cell lysis was alleviated also at the exhaustion of glucose which was evidenced by lesser lysis of the central cells compared to the peripheral cells (Figure 4). These data suggest that glucose exhaustion itself is not a trigger of the colR mutant lysis; rather, this mutant cannot respond adequately to a certain glucose concentration range which finally causes cell death. This scenario also allows to explain the absence of the lysis phenotype in liquid glucose medium. Obviously, the period of nutrient limitation is transient in liquid batch culture and could have been easily missed in our experiments. Literature data suggest that bacteria growing under suboptimal levels of nutrient, i.e. under conditions between the feast and the famine, express cellular responses that are significantly different from those of rapid growth and starvation $[3,48]$. Under conditions of hunger when a nutrient becomes limiting but is not yet depleted, bacteria increase permeability of the membrane to facilitate nutrient entry. For instance, a significantly increased expression of the OprF porin and the LamB-Mgl high-affinity glucose uptake system is considered to be the hunger response of $E$. coli under glucose 
limitation [5]. Analogously, we detected essential nutrient concentration-dependent changes in the OM protein composition of the glucose-grown $P$. putida. We found that the abundance of the sugar channel OprB1 was significantly increased and that of OprE was drastically decreased under low glucose concentrations (Figure 6A). Interestingly, in addition to being modulated by glucose, the abundance of OprE also responds to anaerobiosis $[54,55]$ suggesting that this outer membrane channel contributes to the adaptation to various environmental conditions. OprB1 is known to mediate high-affinity glucose transport both in $P$. putida and $P$. aeruginosa $[25,56,57]$. While OprB1 is not essential for the glucose transport at higher substrate concentrations, it becomes rate-limiting in nutrient uptake at micromolar (1-10 $\mu \mathrm{M})$ glucose concentrations [25,57]. Therefore, the upregulation of OprB1 at low glucose concentrations can be considered an adaptive response of hungry bacteria to stimulate glucose acquisition. However, our results show that the spatiotemporal expression of OprB1 generates spatiotemporal lethal toxicity for colR-deficient bacteria, which implies that the ColRS two-component system is an essential regulator of the hunger response of the glucose-growing $P$. putida.

Our data demonstrate that the up-regulation of OprB1 in response to hunger is controlled post-transcriptionally and that catabolite repression control (CRC) protein $\mathrm{Crc}$ is one of the factors involved in this regulation (Figure 7). CRC is an important global control system in bacteria allowing hierarchical assimilation of substrates under simultaneous presence of several possible carbon sources. Interestingly, while in many bacteria glucose is a preferred carbon source, Pseudomonas prefers organic acids and amino acids to glucose [51,58]. Recent transcriptomic and proteomic data have revealed that $\mathrm{Crc}$ protein inhibits several glucose transport and metabolism genes when $P$. putida grows in nutrient-rich LB medium [53]. For instance, the inactivation of the $\mathrm{crc}$ gene resulted in three times higher abundance of OprB1 in LB-grown cells [53]. Interestingly, it was recently reported that $\mathrm{Crc}$ is not important for the growth of $P$. putida DOT-T1E on glucose as single carbon source and this was explained by dispensability of $\mathrm{Crc}$ in the medium lacking nutrients alternative to glucose [52]. However, our data demonstrate that Crc can actually affect the usage of glucose as the sole carbon source because the abundance of OprB1 was shown to be elevated in the $\operatorname{crc}$ mutant. Yet, the effect of Crc on the amount of OprB1 was observed only in glucose-rich but not in glucose-limiting conditions (Figure 7D) suggesting that the Crc-mediated repression of OprB1 is probably completely absent in hungry bacteria allowing a full expression of OprB1. Thus, in addition to regulating the hierarchical use of carbon sources in complete medium,
Crc is also involved in fine tuning single carbon source assimilation.

The up-regulation of the glucose-scavenging OprB1 is the most appropriate behavior of P. putida at glucose limitation. However, "there is no free lunch in nature." Data of this study suggest that hunger response is costly and if not regulated properly, it might be even deadly as judged by the requirement of ColRS signaling. Interestingly, a largely similar cell death phenomenon was recently characterized in $E$. coli where constitutive expression of the maltoporin LamB resulted in cell lysis in the absence of a functional response regulator OmpR $[59,60]$. The authors proposed that cell death resulted from envelope stress involving an imbalance in the lipopolysaccharide/porin composition of the outer membrane and an increased requirement for inorganic phosphate [60]. Analogous scenario can be considered for the colR mutant, as recent studies conducted in $P$. fluorescens and Xanthomonas citri have indicated that ColRS system is involved in LPS production and/or modification [20,61].

Our current study describes not only the participation of ColRS system in hunger response of $P$. putida, but also provides clues to better understand the role of this system in root colonization. It is notable that the colonization defect observed for P. fluorescens ColRS system mutant became evident only under the condition of competition with the wild-type strain [19]. This indicates that the colonization ability per se is not impaired but rather some other population-related trait is hampered in the absence of ColRS signaling. Our results suggest that hunger-induced lysis of a subpopulation may be responsible for the reduced fitness of the colR mutant under competition conditions. Nutrient concentration in the rhizosphere is low [62] and thereby rhizosphere colonization takes place under condition of hunger [63]. We assume that nutrient limitation may result in the hunger-induced subpopulation lysis of the colR mutant and this obviously will reduce the fitness of colR mutant bacteria in co-cultivation experiments. However, if the colR mutant grows as a pure culture, its colonization ability is not affected because nutrients liberated from lysed cells probably support the growth of surviving population. In the future, it would be very interesting to examine the impact of the ColRS system on the viability of different Pseudomonas species in the rhizosphere.

\section{Conclusions}

Current study demonstrated that the glucose-growing $P$. putida responds to a low glucose level by the up-regulation of the sugar channel OprB1, which most probably facilitates nutrient scavenging under hunger conditions (Figure 8). We present evidence that on the glucose-rich 


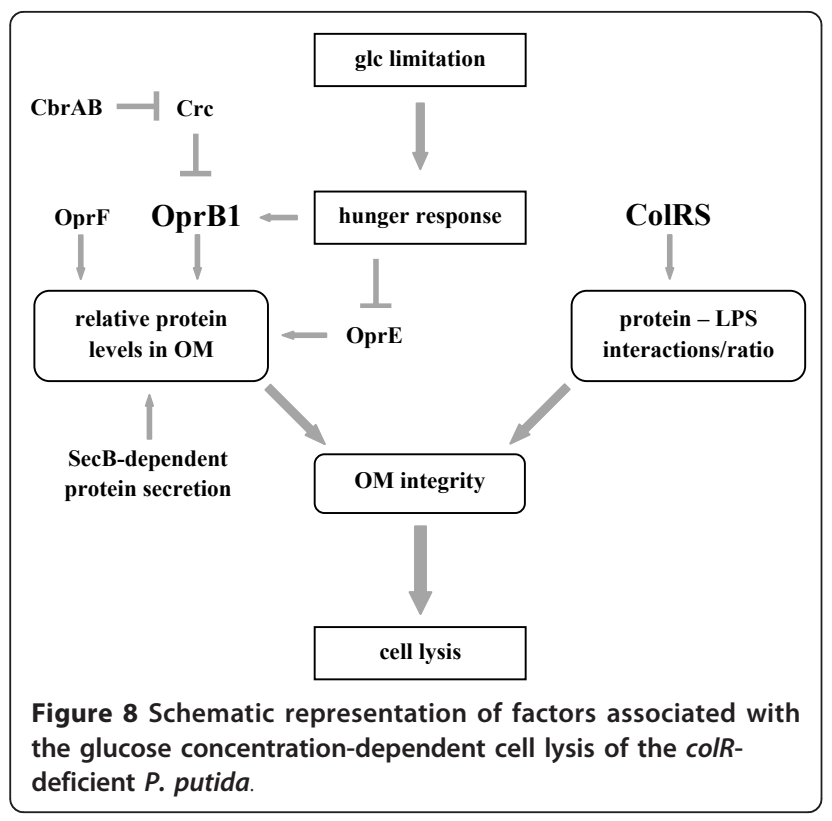

medium the OprB1 expression is post-transcriptionally repressed, and carbon catabolite repression regulator $\mathrm{Crc}$ is partially responsible for that. Most interestingly, we show that the hunger-induced expression of OprB1 is lethal to bacteria deficient in ColR as deduced from a clear correlation between the amount of OprB1 and the cell death of the colR mutant. However, the glucose-induced death of the colR mutant can be suppressed by reducing the abundance of various membrane proteins such as the OprB1 and OprF as well as excluding the SecB-dependent protein secretion (Figure 8). Thus, the ColRS system could be considered a safety factor of hunger response as it ensures the welfare of cell membrane during increased synthesis of certain membrane proteins.

\section{Acknowledgements}

We are grateful to Niilo Kaldalu for fruitful discussions and advice. We thank Tiina Alamäe, Hiie Saumaa, Maia Kivisaar, Paula Ann Kivistik, and Hanna Hõrak for their critical reading of the manuscript. We thank Riho Teras for plasmid pUCNotKm, Olga Šapran for the assistance in cloning, and Liisa Arike for protein identification. Mass spectrometric analyses were supported in part by the European Regional Development Fund through the Center of Excellence in Chemical Biology (Institute of Technology, University of Tartu). This work was supported by the grant 7829 from the Estonian Science Foundation and by Targeted Financing Project TLOMRO031 from the Estonian Ministry of Research and Education.

\section{Authors' contributions}

MP and RH prepared design of experimental work. MP carried out transposon mutagenesis screen and participated in OMP analysis. AA purified OMPs and did OMP pattern analysis. HI constructed mutant strains and contributed enzyme assays. RH performed lysis assays, coordinated experimental work and wrote the manuscript. All authors participated in manuscript editing and approved the final manuscript.

Received: 17 February 2011 Accepted: 26 July 2011 Published: 26 July 2011

\section{References}

1. Navarro Llorens JM, Tormo A, Martinez-Garcia E: Stationary phase in gramnegative bacteria. FEMS Microbiol Rev 2010, 34(4):476-495.

2. Ferenci T: Bacterial physiology, regulation and mutational adaptation in a chemostat environment. Adv Microb Physiol 2008, 53:169-229.

3. Ferenci T: Hungry bacteria-definition and properties of a nutritional state. Environ Microbiol 2001, 3(10):605-611.

4. Harder W, Dijkhuizen L: Physiological responses to nutrient limitation. Annu Rev Microbiol 1983, 37:1-23.

5. Liu X, Ferenci T: Regulation of porin-mediated outer membrane permeability by nutrient limitation in Escherichia coli. J Bacterio/ 1998, 180(15):3917-3922

6. Liu X, Ferenci T: An analysis of multifactorial influences on the transcriptional control of $o m p F$ and $o m p C$ porin expression under nutrient limitation. Microbiology 2001, 147:2981-2989.

7. Death A, Notley L, Ferenci T: Derepression of LamB protein facilitates outer membrane permeation of carbohydrates into Escherichia coli under conditions of nutrient stress. J Bacteriol 1993, 175(5):1475-1483.

8. Hua Q, Yang C, Oshima T, Mori H, Shimizu K: Analysis of gene expression in Escherichia coli in response to changes of growth-limiting nutrient in chemostat cultures. Appl Environ Microbiol 2004, 70(4):2354-2366.

9. Death A, Ferenci T: The importance of the binding-protein-dependent $\mathrm{Mgl}$ system to the transport of glucose in Escherichia coli growing on low sugar concentrations. Res Microbiol 1993, 144(7):529-537.

10. Shapiro JA: Thinking about bacterial populations as multicellular organisms. Annu Rev Microbiol 1998, 52:81-104.

11. Salhi $\mathrm{B}$, Mendelson NH: Patterns of gene expression in Bacillus subtilis colonies. J Bacteriol 1993, 175(16):5000-5008.

12. Mendelson NH, Salhi B: Patterns of reporter gene expression in the phase diagram of Bacillus subtilis colony forms. J Bacteriol 1996, 178(7):1980-1989.

13. Stewart PS, Franklin MJ: Physiological heterogeneity in biofilms. Nat Rev Microbiol 2008, 6(3):199-210.

14. Pamp SJ, Gjermansen M, Johansen HK, Tolker-Nielsen T: Tolerance to the antimicrobial peptide colistin in Pseudomonas aeruginosa biofilms is linked to metabolically active cells, and depends on the pmr and mexAB-oprM genes. Mol Microbiol 2008, 68(1):223-240.

15. Espinosa-Urgel M, Kolter R, Ramos JL: Root colonization by Pseudomonas putida: love at first sight. Microbiology 2002, 148:341-343.

16. Timmis KN: Pseudomonas putida: a cosmopolitan opportunist par excellence. Environ Microbiol 2002, 4(12):779-781.

17. Wu X, Monchy S, Taghavi S, Zhu W, Ramos J, van der Lelie D: Comparative genomics and functional analysis of niche-specific adaptation in Pseudomonas putida. FEMS Microbiol Rev 2010, 35(2):299-323.

18. Winsor GL, Van Rossum T, Lo R, Khaira B, Whiteside MD, Hancock RE, Brinkman FS: Pseudomonas Genome Database: facilitating user-friendly, comprehensive comparisons of microbial genomes. Nucleic Acids Res 2009, 37:D483-488

19. Dekkers LC, Bloemendaal CJ, de Weger LA, Wijfelman CA, Spaink HP, Lugtenberg BJ: A two-component system plays an important role in the root-colonizing ability of Pseudomonas fluorescens strain WCS365. Mol Plant Microbe Interact 1998, 11(1):45-56.

20. de Weert S, Dekkers LC, Bitter W, Tuinman S, Wijjjes AH, van Boxtel R, Lugtenberg BJ: The two-component colR/S system of Pseudomonas fluorescens WCS365 plays a role in rhizosphere competence through maintaining the structure and function of the outer membrane. FEMS Microbiol Ecol 2006, 58(2):205-213.

21. Garvis S, Munder A, Ball G, de Bentzmann S, Wiehlmann L, Ewbank JJ, Tümmler B, Filloux A: Caenorhabditis elegans semi-automated liquid screen reveals a specialized role for the chemotaxis gene cheB2 in Pseudomonas aeruginosa virulence. PLoS Pathog 2009, 5(8):e1000540

22. Hõrak R, Ilves $H$, Pruunsild P, Kuljus M, Kivisaar M: The ColR-ColS twocomponent signal transduction system is involved in regulation of Tn4652 transposition in Pseudomonas putida under starvation conditions. Mol Microbiol 2004, 54(3):795-807.

23. Kivistik PA, Putrinš $M$, Püvi $K$, Ilves $H$, Kivisaar $M$, Hõrak R: The ColRS twocomponent system regulates membrane functions and protects Pseudomonas putida against phenol. J Bacterio/ 2006, 188(23):8109-8117.

24. Hu N, Zhao B: Key genes involved in heavy-metal resistance in Pseudomonas putida CD2. FEMS Microbiol Lett 2007, 267(1):17-22. 
25. Putrinš $M$, Ilves $H$, Kivisaar $M$, Hõrak R: ColRS two-component system prevents lysis of subpopulation of glucose-grown Pseudomonas putida. Environ Microbiol 2008, 10(10):2886-2893.

26. Bayley SA, Duggleby CJ, Worsey MJ, Williams PA, Hardy KG, Broda P: Two modes of loss of the Tol function from Pseudomonas putida mt-2. Mol Gen Genet 1977, 154(2):203-204.

27. Regenhardt D, Heuer H, Heim S, Fernandez DU, Strömpl C, Moore ER, Timmis KN: Pedigree and taxonomic credentials of Pseudomonas putida strain KT2440. Environ Microbiol 2002, 4(12):912-915.

28. Miller $\mathrm{JH}$ : A short course in bacterial genetics: a laboratory manual and handbook for Echerichia coli and related bacteria. Cold Spring Harbour Laboratory Press, Cold Spring Harbour, NY; 1992.

29. Adams MH: Bacteriophages. Interscience Publishers Inc., New York; 1959.

30. Sharma RC, Schimke RT: Preparation of electrocompetent E. coli using salt-free growth medium. Biotechniques 1996, 20(1):42-44.

31. O'Toole GA, Kolter R: Initiation of biofilm formation in Pseudomonas fluorescens WCS365 proceeds via multiple, convergent signalling pathways: a genetic analysis. Mol Microbiol 1998, 28(3):449-461.

32. Yuste L, Rojo F: Role of the crc gene in catabolic repression of the Pseudomonas putida GPo1 alkane degradation pathway. J Bacteriol 2001, 183(21):6197-6206

33. Tover A, Ojangu EL, Kivisaar M: Growth medium composition-determined regulatory mechanisms are superimposed on CatR-mediated transcription from the pheBA and catBCA promoters in Pseudomonas putida. Microbiology 2001, 147:2149-2156.

34. Putrinš $M$, Ilves $H$, Lilje L, Kivisaar M, Hõrak R: The impact of ColRS twocomponent system and $\operatorname{Ttg} A B C$ efflux pump on phenol tolerance of Pseudomonas putida becomes evident only in growing bacteria. BMC Microbiol 2010, 10:110.

35. Hõrak R, Kivisaar M: Expression of the transposase gene tnpA of Tn4652 is positively affected by integration host factor. J Bacteriol 1998, 180(11):2822-2829.

36. Shevchenko A, Tomas H, Havlis J, Olsen JV, Mann M: In-gel digestion for mass spectrometric characterization of proteins and proteomes. Nat Protoc 2006, 1(6):2856-2860.

37. Rappsilber J, Mann M, Ishihama Y: Protocol for micro-purification, enrichment, pre-fractionation and storage of peptides for proteomics using StageTips. Nat Protoc 2007, 2(8):1896-1906.

38. Olsen JV, de Godoy LM, Li G, Macek B, Mortensen P, Pesch R, Makarov A, Lange $\mathrm{O}$, Horning S, Mann M: Parts per million mass accuracy on an Orbitrap mass spectrometer via lock mass injection into a C-trap. $\mathrm{Mol}$ Cell Proteomics 2005, 4(12):2010-2021.

39. Nishijyo T, Haas D, Itoh $Y$ : The CbrA-CbrB two-component regulatory system controls the utilization of multiple carbon and nitrogen sources in Pseudomonas aeruginosa. Mol Microbiol 2001, 40(4):917-931.

40. Zhang XX, Rainey PB: Dual involvement of CbrAB and NtrBC in the regulation of histidine utilization in Pseudomonas fluorescens SBW25. Genetics 2008, 178(1):185-195.

41. Brinkman FS, Schoofs G, Hancock RE, De Mot R: Influence of a putative ECF sigma factor on expression of the major outer membrane protein, OprF, in Pseudomonas aeruginosa and Pseudomonas fluorescens. J Bacteriol 1999, 181(16):4746-4754.

42. Driessen AJ, Nouwen N: Protein translocation across the bacterial cytoplasmic membrane. Annu Rev Biochem 2008, 77:643-667.

43. Fekkes $P$, van der Does $C$, Driessen AJ: The molecular chaperone SecB is released from the carboxy-terminus of SecA during initiation of precursor protein translocation. Embo J 1997, 16(20):6105-6113.

44. van Wely KH, Swaving J, Klein M, Freudl R, Driessen AJ: The carboxyl terminus of the Bacillus subtilis SecA is dispensable for protein secretion and viability. Microbiology 2000, 146:2573-2581.

45. Hancock RE, Carey AM: Outer membrane of Pseudomonas aeruginosa: heat- 2-mercaptoethanol-modifiable proteins. J Bacteriol 1979, 140(3):902-910.

46. del Castillo T, Ramos JL, Rodriguez-Herva JJ, Fuhrer T, Sauer U, Duque E: Convergent peripheral pathways catalyze initial glucose catabolism in Pseudomonas putida: genomic and flux analysis. J Bacteriol 2007, 189(14):5142-5152.

47. Saravolac EG, Taylor NF, Benz R, Hancock RE: Purification of glucoseinducible outer membrane protein OprB of Pseudomonas putida and reconstitution of glucose-specific pores. J Bacteriol 1991, 173(16):4970-4976.
48. Ferenci T: Regulation by nutrient limitation. Curr Opin Microbiol 1999, 2(2):208-213.

49. Sonnleitner E, Abdou L, Haas D: Small RNA as global regulator of carbon catabolite repression in Pseudomonas aeruginosa. Proc Natl Acad Sci USA 2009, 106(51):21866-21871.

50. Moreno R, Marzi S, Romby P, Rojo F: The Crc global regulator binds to an unpaired A-rich motif at the Pseudomonas putida alkS mRNA coding sequence and inhibits translation initiation. Nucleic Acids Res 2009, 37(22):7678-7690.

51. Rojo F: Carbon catabolite repression in Pseudomonas : optimizing metabolic versatility and interactions with the environment. FEMS Microbiol Rev 2010, 34(5):658-684.

52. Daniels C, Godoy P, Duque E, Molina-Henares MA, de la Torre J, Del Arco JM, Herrera C, Segura A, Guazzaroni ME, Ferrer M, Ramos JL: Global regulation of food supply by Pseudomonas putida DOT-T1E. J Bacteriol 2010, 192(8):2169-2181.

53. Moreno R, Martinez-Gomariz M, Yuste L, Gil C, Rojo F: The Pseudomonas putida $\mathrm{Crc}$ global regulator controls the hierarchical assimilation of amino acids in a complete medium: evidence from proteomic and genomic analyses. Proteomics 2009, 9(11):2910-2928.

54. Jaouen T, Coquet L, Marvin-Guy L, Orange N, Chevalier S, De E: Functional characterization of Pseudomonas fluorescens OprE and OprQ membrane proteins. Biochem Biophys Res Commun 2006, 346(3):1048-1052.

55. Yamano Y, Nishikawa T, Komatsu Y: Cloning and nucleotide sequence of anaerobically induced porin protein E1 (OprE) of Pseudomonas aeruginosa PAO1. Mol Microbiol 1993, 8(5):993-1004.

56. Shrivastava R, Basu B, Godbole A, Mathew MK, Apte SK, Phale PS: Repression of the glucose-inducible outer-membrane protein OprB during utilization of aromatic compounds and organic acids in Pseudomonas putida CSV86. Microbiology 2011, 157:1531-1540.

57. Wylie $\lrcorner$, Worobec EA: The OprB porin plays a central role in carbohydrate uptake in Pseudomonas aeruginosa. J Bacteriol 1995 177(11):3021-3026.

58. Görke B, Stülke J: Carbon catabolite repression in bacteria: many ways to make the most out of nutrients. Nat Rev Microbio/ 2008, 6(8):613-624.

59. Reimann SA, Wolfe AJ: A critical process controlled by MalT and OmpR is revealed through synthetic lethality. J Bacterio/ 2009, 191(16):5320-5324.

60. Reimann SA, Wolfe AJ: Constitutive Expression of the Maltoporin LamB in the Absence of OmpR Damages the Cell Envelope. J Bacterio/ 2011, 193(4):842-853.

61. Yan Q, Wang N: The ColR/ColS Two-Component System Plays Multiple Roles in the Pathogenicity of the Citrus Canker Pathogen Xanthomonas citri subsp. citri. J Bacteriol 2011, 193(7):1590-1599.

62. Lugtenberg BJ, Kravchenko LV, Simons M: Tomato seed and root exudate sugars: composition, utilization by Pseudomonas biocontrol strains and role in rhizosphere colonization. Environ Microbiol 1999, 1(5):439-446.

63. Lugtenberg B, Kamilova F: Plant-growth-promoting rhizobacteria. Annu Rev Microbiol 2009, 63:541-556.

64. Herrero M, de Lorenzo V, Timmis KN: Transposon vectors containing nonantibiotic resistance selection markers for cloning and stable chromosomal insertion of foreign genes in gram-negative bacteria. $J$ Bacteriol 1990, 172(11):6557-6567.

65. Wilson KJ, Sessitsch A, Corbo JC, Giller KE, Akkermans AD, Jefferson RA: beta-Glucuronidase (GUS) transposons for ecological and genetic studies of rhizobia and other gram-negative bacteria. Microbiology 1995 , 141:1691-1705.

66. Figurski DH, Helinski DR: Replication of an origin-containing derivative of plasmid RK2 dependent on a plasmid function provided in trans. Proc Natl Acad Sci USA 1979, 76(4):1648-1652.

67. Ojangu EL, Tover A, Teras R, Kivisaar M: Effects of combination of different -10 hexamers and downstream sequences on stationary-phase-specific sigma factor sigma(S)-dependent transcription in Pseudomonas putida. J Bacteriol 2000, 182(23):6707-6713.

68. Koch B, Jensen LE, Nybroe $\mathrm{O}$ : A panel of Tn7-based vectors for insertion of the gfp marker gene or for delivery of cloned DNA into Gramnegative bacteria at a neutral chromosomal site. J Microbiol Methods 2001, 45(3):187-195.

doi:10.1186/1471-2180-11-170

Cite this article as: Putrinš et al:: The ColRS system is essential for the hunger response of glucose-growing Pseudomonas putida. BMC Microbiology 2011 11:170. 